\title{
Edaravone-Encapsulated Agonistic Micelles Rescue Ischemic Brain Tissue by Tuning Blood-Brain Barrier Permeability
}

\author{
Qu Jin ${ }^{*}$, Yu Cai ${ }^{*}$, Sihan $\mathrm{Li}^{1}$, Haoran Liu${ }^{1}$, Xingyu Zhou ${ }^{1}$, Chunqiang Lu², Xihui Gao ${ }^{1}$, Jun Qian ${ }^{1}$, Jun \\ Zhang ${ }^{3}$, Shenghong Ju ${ }^{2 \bowtie}$ and Cong $\mathrm{Li}^{1 \bowtie}$ \\ 1. Key Laboratory of Smart Drug Delivery, Ministry of Education, School of Pharmacy, Fudan University, 826 Zhangheng Road, Shanghai 201203, P. R. China. \\ 2. Jiangsu Key Laboratory of Molecular and Functional Imaging Department of Radiology, Zhongda Hospital, Medical School of Southeast University, 87 \\ Dingjiaqiao Road, Nanjing 210009, P. R. China. \\ 3. Department of Radiology, Huashan Hospital, Fudan University, 12 Wulumuqi Middle Road, Shanghai 200041, P. R. China. \\ *These authors share equal first authorship. \\ $\bowtie$ Corresponding authors: Shenghong Ju, Email: jsh0836@hotmail.com; Cong Li, Email: congli@fudan.edu.cn. \\ (C) Ivyspring International Publisher. This is an open access article distributed under the terms of the Creative Commons Attribution (CC BY-NC) license \\ (https://creativecommons.org/licenses/by-nc/4.0/). See http://ivyspring.com/terms for full terms and conditions.
}

Received: 2016.11.05; Accepted: 2016.11.23; Published: 2017.02.08

\begin{abstract}
Thrombolysis has been a standard treatment for ischemic stroke. However, only 2-7\% patients benefit from it because the thrombolytic agent has to be injected within $4.5 \mathrm{~h}$ after the onset of symptoms to avoid the increasing risk of intracerebral hemorrhage. As the only clinically approved neuroprotective drug, edaravone (EDV) rescues ischemic brain tissues by eradicating over-produced reactive oxygen species (ROS) without the limitation of therapeutic time-window. However, EDV's short circulation half-life and inadequate cerebral uptake attenuate its therapeutic efficacy. Here we developed an EDV-encapsulated agonistic micelle (EDV-AM) to specifically deliver EDV into brain ischemia by actively tuning blood-brain barrier (BBB) permeability. The EDV-AM actively up-regulated endothelial monolayer permeability in vitro. HPLC studies showed that EDV-AM delivered more EDV into brain ischemia than free EDV after intravenous injection. Magnetic resonance imaging also demonstrated that EDV-AM more rapidly salvaged ischemic tissue than free EDV. Diffusion tensor imaging indicated the highest efficiency of EDV-AM in accelerating axonal remodeling in the ipsilesional white matter and improving functional behaviors of ischemic stroke models. The agonistic micelle holds promise to improve the therapeutic efficiency of ischemic stroke patients who miss the thrombolytic treatment.
\end{abstract}

Key words: ischemic stroke, blood-brain-barrier, neuroprotective therapy, agonistic micelle, edaravone.

\section{Introduction}

Ischemic stroke is triggered by a reduction in cerebral blood flow (CBF) caused either by in situ thrombosis or embolization of a clot from a proximal arterial or cardiac source [1]. It accounts for approximately $85 \%$ of all cases of stroke - the second major cause of death and a leading cause of adult disability worldwide [2]. Recombinant tissue plasminogen activator (rt-PA) is the only FDA-approved thrombolytic agent that aims to break down vascular occlusions and rapidly restores the CBF [3]. Even though rt-PA mediated thrombolysis has been used as a standard treatment for ischemic stroke [4], only a few patients (2-7\%) benefit from it because this drug must be injected within $4.5 \mathrm{~h}$ post onset of stroke symptom to prevent intracerebral hemorrhage [5]. Therefore, it is critical to develop novel therapeutic strategies to treat the majority of ischemic stroke patients who miss the time-window of rt-PA treatment.

As a promising treatment strategy, neuroprotection aims to improve the survival of brain tissues by impeding damaging ischemic cascade events including excessive activation of glutamate receptors, an influx of calcium cations, 
over-production of free radicals and abnormal recruitment of inflammatory cells. Reactive oxygen species (ROS) are believed to play a pivotal role in neuronal damage after ischemic stroke [6]. First, the brain is the most vulnerable organ to ROS due to its high oxygen consumption, lack of endogenous antioxidants and high concentration of peroxidisable lipids [7]. Second, ROS damage intracerebral vasculatures and result in fatal consequences such as vasogenic edema and hemorrhagic transformation [8]. Third, over-production of ROS and their destructive effects are observed in hyperacute (hours), subacute (days) and chronic stages (weeks to months) [9]. Therefore, a neuroprotective strategy eradicating ROS efficiently and persistently will be beneficial to patients who fail the thrombolytic treatment due to the critical time window.

Edaravone (EDV) is a small molecular ROS scavenger that was approved for the treatment of ischemic stroke patients in Japan in 2001 [10]. EDV reduce ischemic lesion sizes in patients by decreasing oxidative stress, inhibiting lipid peroxidation and attenuating long-term inflammation [11]. Even though early studies suggest that EDV should be administered in the hyperacute stage to maximize the neuroprotective effect [12], recent work showed the neuroprotective benefits to the patients even when this ROS scavenger was injected as long as $24 \mathrm{~h}$ after the stroke onset [13]. Due to EDV's short half-life (5.4 $\mathrm{min})$, a relatively high dose (30 $\mathrm{mg}$ twice/day/person) had to be injected which resulted in complications such as renal function disorder [14]. Previous studies showed the synergistic neuroprotective effects after the combined treatment of EDV and borneol in ischemic stroke models [15]. Considering the capability of borneol to increase blood-brain-barrier (BBB) permeability [16], we hypothesize that the neuroprotective effect of EDV could be amplified by specifically enhancing its uptake in brain ischemia. EDV-loaded liposomes demonstrated higher therapeutic response than free EDV in the treatment of light-induced retinal dysfunction by eyedrop administration [17]. Therefore, we believe that specifically delivering EDV into brain ischemia by a BBB-permeable nanovector may be a promising way to treat ischemic stroke with high efficiency and extended intervention time-window.

As a unique physiological structure in brain vasculature, the BBB separates the central nervous system (CNS) from the periphery circulation and creates a precisely controlled environment for major brain circuit tasks such as proper functional connectivity, synaptic transmission and information processing [18]. Due to its low permeability, the majority of small molecular drugs and nearly all the macromolecular drugs cannot enter the brain parenchyma [19]. Even though pathological BBB permeability enhancement was observed in brain ischemia, therapeutic effects of neuroprotective drugs such as brain-derived neurotrophic factor (BDNF) were not prominent because their cerebral concentrations were still too low to meet the therapeutic threshold [20]. Recently, we reported a dendrimer-based nanoagonist that tuned BBB permeability by signaling adenosine $2 \mathrm{~A}$ receptor $\left(\mathrm{A}_{2 \mathrm{~A}} \mathrm{R}\right)$, a subtype of purinergic $\mathrm{G}$ protein-coupled receptors (GPCRs) expressed on the luminal side of brain capillaries [21]. Pre-injection of nanoagonist efficiently increased cerebral uptake and neuroprotective effect of ROS scavenger superoxide dismutase (SOD) that was injected when the BBB permeability was maximum by magnetic resonance imaging (MRI) [22]. However, the two-step injection strategy faces hurdles for clinical translation due to the technical inconvenience, prolonged anesthetic time, and requirement of complicated imaging facilities. Therefore, there is an urgent need to develop vectors to simultaneously enhance BBB permeability and deliver the neuroprotectant into brain ischemia.

In this study, we developed an agonistic micelle (EDV-AM) to specifically deliver EDV into brain ischemia by actively opening tight junctions (TJs) that are major structures in regulating BBB permeability by strictly limiting para-endothelial movements of liquids, ions, and molecules [23, 24]. The EDV is encapsulated in the hydrophobic inner core of the micelle with a high payload, while its hydrophilic outer shell is modified with $\mathrm{A}_{2 \mathrm{~A}} \mathrm{R}$ agonistic agents and near-infrared (NIR) fluorophores. Due to the up-regulated $A_{2 A} R$ in brain capillaries of the ischemic region [22], EDV-AM will first target the ischemic vasculatures. EDV-AM - $\mathrm{A}_{2 \mathrm{~A}} \mathrm{R}$ signaling will trigger TJ opening and facilitate its specific delivery into brain ischemia (Figure 1). EDV released from intracerebral EDV-AM efficiently eradicates ROS produced by resident brain cells (mainly microglia) as well as the infiltrated inflammatory cells (including macrophages, neutrophils, and $\mathrm{T}$ cells). Also, the rapid restoration of the TJs will minimize side effects caused by irreversible BBB breakdown. To our knowledge, EDV-AM is the first nanovector for the treatment of ischemic stroke by actively tuning BBB permeability. These agonistic micelles hold promise for conferring neuroprotective effect by sustainably eliminating intracerebral ROS. This will be beneficial to the majority of ischemic stroke patients who miss the short intervention time-window for thrombolytic therapy. 


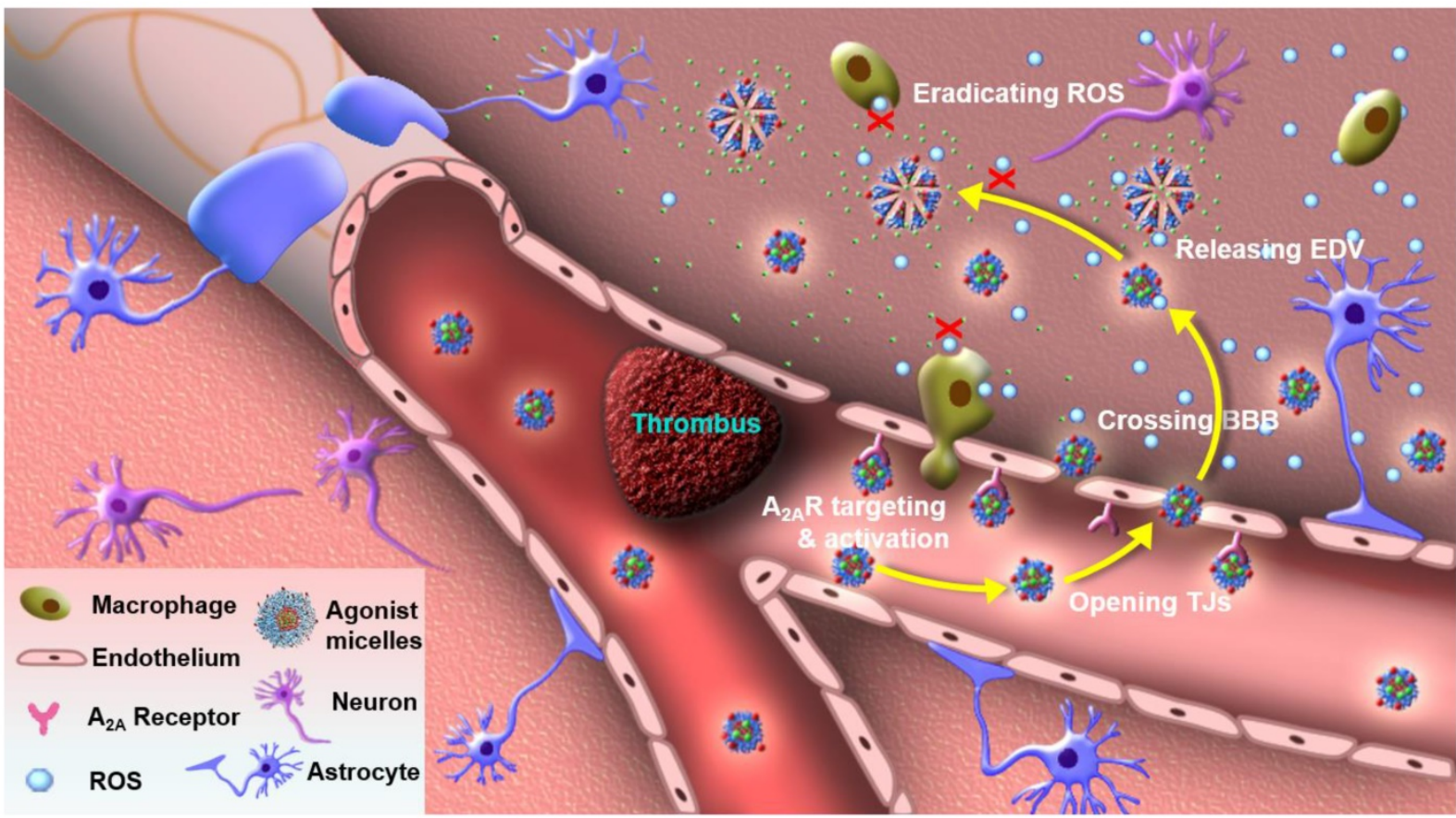

Figure 1. Agonistic micelles salvage brain ischemic tissue. EDV-AM specifically activated $A_{2 A} R$ over-expressed in the capillaries of brain ischemia, increased $B B B$ permeability by temporarily opening para-endothelial TJs, entered brain ischemia by crossing the compromised T]s, released the encapsulated EDV, and sustainably eradicated ROS excreted by brain-derived cells (microglia, astrocytes and endothelium) as well as infiltrated inflammatory cells (macrophages and neutrophils).

\section{Results \& Discussion}

\section{Up-regulated A2AR Level in Ischemic Brain Vasculatures}

Up-regulated expression of $\mathrm{A}_{2 \mathrm{~A}} \mathrm{R}$ in ischemic vasculatures is a prerequisite for specifically tuning BBB permeability by the agonistic micelles. As shown in Figure 2A, $A_{2 A} R$ immune activity in ipsilateral hemisphere (I.H.) was evident and located in brain capillaries immunostained with the CD31 antibody. In contrast, $\mathrm{A}_{2 \mathrm{~A}} \mathrm{R}$ expression was not apparent on the capillaries in the contralateral hemisphere (C.H.). The average perivascular $A_{2 A} R$ immunofluorescence intensity in I.H. was determined to be 3.7 times higher than that in C.H. (Figure 2B). Previous immunoblotting studies showed that the $\mathrm{A}_{2 \mathrm{~A}} \mathrm{R}$ expression level in bEnd.3 endothelial cells cultured under hypoxia was $72 \%$ higher than that cultured under normoxia [22]. Present and previous studies indicate the possibility to specifically modulate BBB permeability in ischemic regions by simultaneously targeting and signaling the over-expressed $\mathrm{A}_{2 \mathrm{~A}} \mathrm{R}$.

\section{Design, synthesis, and characterization of agonist micelles}

Amphiphilic copolymer methoxypoly (ethylene glycol)-b-poly (D,L-lactic acid) (PEG-PLA) was chosen to develop the micelles due to: (1) the convenience to encapsulate EDV with high payload in its hydrophobic core and to label agonistic agents on its hydrophilic shell, (2) nano-range particle diameter (20-100 nm) facilitating its brain uptake by crossing the compromised TJs, (3) improved drug bioavailability due to the prolonged circulation time and well preserved activity [25]. Even though several PEG-PLA micelle-based anticancer drugs such as Genexol-PM have been approved for clinical trials in Korea, UK, and the USA [26], no micelle-based neuroprotective drugs for ischemic stroke treatment have yet been reported. Purine nucleotide derivative CGS21680 was chosen as both an $\mathrm{A}_{2 \mathrm{~A}} \mathrm{R}$ targeting and signaling agent due to its high binding affinity $\left(\mathrm{K}_{\mathrm{d}}=\right.$ $15.5 \mathrm{nM}$ ) and specificity to $\mathrm{A}_{2 \mathrm{~A}} \mathrm{R}$ (10-fold against $\mathrm{A}_{1} \mathrm{R}$ and $>13,300$-fold against $A_{2 B} R$ ) [27]. Compared to the previously reported pluripotent nanoparticles [28], CGS21680, as both a targeting and agonistic agent, not only simplifies the synthetic procedure but is also helpful in preparing micelles with high standardization. Heptamethine cyanine derivative IR783B was chosen as a fluorophore because its near-infrared (NIR) fluorescence can non-invasively track the intracerebral drug delivery [28]. EDV-AM and control micelle EDV-PM without the agonistic domain were developed through an emulsion/solvent evaporation strategy. Self-assembly of PEG-PLA derivatives including MeO-PEG-PLA, IR783B-PEG-PLA, and CGS-PEG-PLA in the presence of EDV resulted in EDV-AM (Figure 2C). Our previous study showed that the TJ opening efficiency and time-window can be tuned by changing the number of agonistic agents labeled on the 
nanoparticles [21]. Therefore, it would be feasible to specifically and efficiently increase BBB permeability in ischemic regions by optimizing the labeling degree of CGS21680 by simply changing the molar percentage of CGS-PEG-PLA in the PEG-PLA building blocks. Transmission electron microscope (TEM) images showed a scattered distribution of EDV-AM micelles as nanospheres with an average diameter of $20 \mathrm{~nm}$ (Figure 2D). The hydrodynamic diameters of EDV-AM and EDV-PM were measured as 23 and $21 \mathrm{~nm}$ with corresponding polydispersity values of 0.124 and 0.106 and their zeta potentials were determined to be -1.8 and $-1.1 \mathrm{mV}$, respectively, by dynamic light scattering (DLS) (Figure 2E and Figure S1). The EDV encapsulation ratio of EDV-AM and EDV-PM were $10.9 \pm 0.1 \%$ and $9.7 \pm 0.1 \%$ and the EDV drug loading values were measured as $3.5 \pm$ $0.05 \%$ and $2.7 \pm 1.00 \%$, respectively. Furthermore, their critical micelle concentrations (CMC) were measured to be $3.9 \mu \mathrm{g} / \mathrm{mL}$ and $6.6 \mu \mathrm{g} / \mathrm{mL}$ respectively, which are comparable to that of PEG-PLA-based micelles $(4.5 \mu \mathrm{g} / \mathrm{mL})$ [29], and indicate their good stability in the blood stream. The physical parameters of the EDV-AM and EDV-PM are summarized in Table $\mathbf{1 .}$
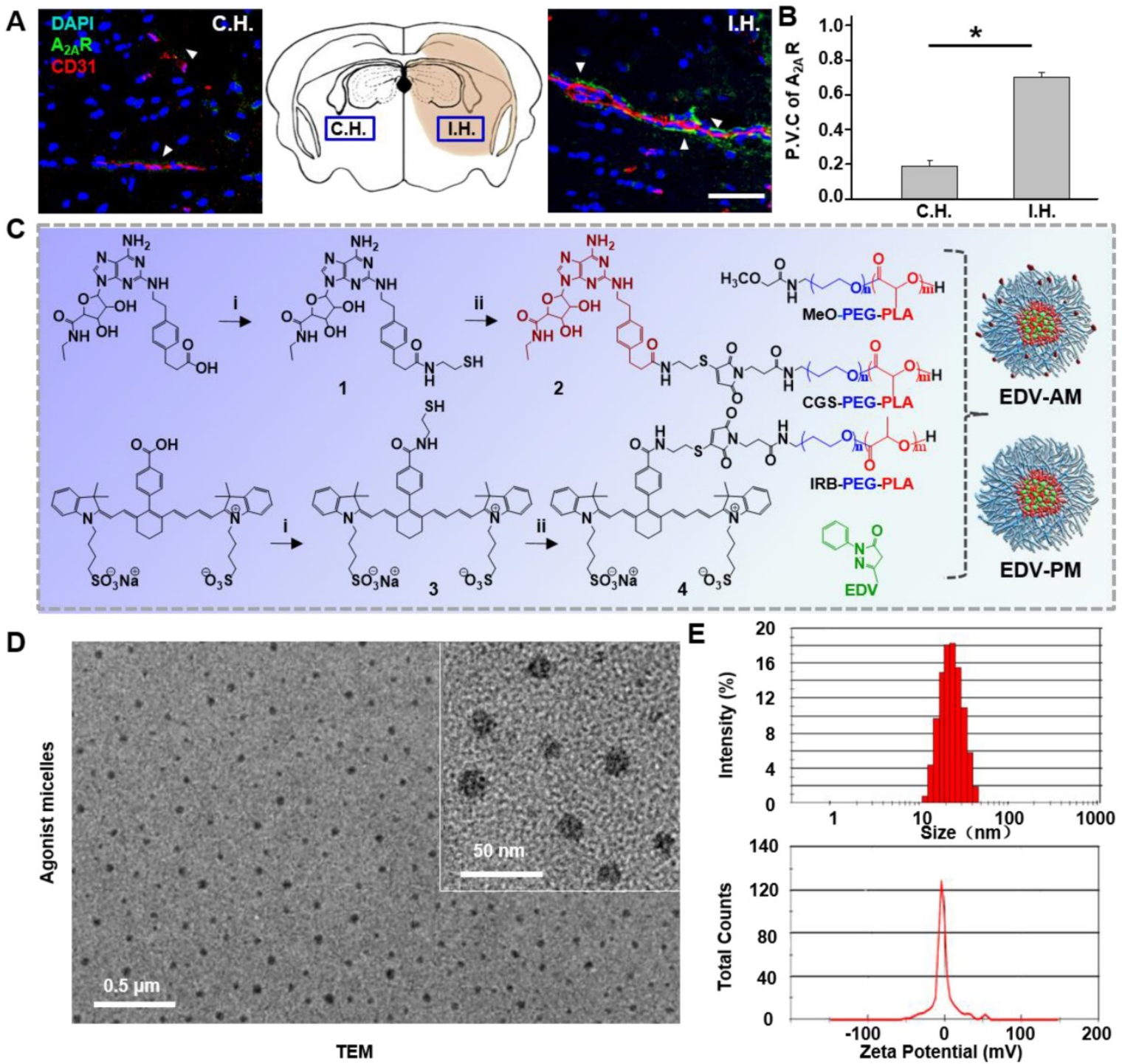

Figure 2. Synthesis and characterization of agonistic micelles. (A) Doubly immunostained microscopic fluorescence images in brain ipsilateral and contralateral striatum at $24 \mathrm{~h}$ post-stroke attack. Immunofluorescence of vascular endothelium and $A_{2 A} R$ are displayed in red and green respectively, the nuclei stained with DAPI is in blue. Scale bar, $100 \mu \mathrm{m}$. (B) The perivascular coverage of $A_{2 A} R$ in the ipsilateral and contralateral striatum. Data are present as mean $\pm S D$ ( $n=16$ slices from 4 animal models), $* P<0.05$. (C) Synthesis of the PEG-PLA copolymer components, EDV-AM and EDV-PM. (D) TEM image of EDV-AM. (E) Dynamic particle size distribution and zeta potential of EDV-AM. C.H.: contralateral hemisphere, I.H.: ipsilateral hemisphere, P.V.C.: perivascular coverage. 
Table 1. Physical parameters of EDV encapsulated micelles.

\begin{tabular}{|c|c|c|c|c|c|c|}
\hline Micelles & $\left.\mathrm{d}(\mathrm{nm})^{\mathrm{a}}\right)$ & PDIa) & $\zeta(\mathrm{mV})^{\mathrm{a})}$ & $\left.\mathrm{CMC}(\mu \mathrm{g} / \mathrm{mL})^{\mathrm{b}}\right)$ & $\mathrm{LRc})$ & ERc) \\
\hline EDV-PM & 21 & 0.106 & -1.1 & 6.57 & $2.7 \pm 1.0 \%$ & $9.7 \pm 0.1 \%$ \\
\hline EDV-AM & 23 & 0.124 & -1.8 & 3.85 & $3.5 \pm 0.05 \%$ & $10.9 \pm 0.1 \%$ \\
\hline
\end{tabular}

Diameter, polydispersity index (PDI), and zeta potential $(\zeta)$ of the micelles were measured by dynamic light scattering (DLS) in PBS (pH 7.4). b) The critical micelles

concentration (CMC) was measured by a UV-visible spectrophotometer. c) The entrapment ratio (ER) and Drug loading ratio (LR) were determined by high-performance liquid chromatography (HPLC). The mobile phase was methanol/0.05 $\mathrm{M}$ ammonium dihydrogen phosphate solution $(\mathrm{pH}=3.5)(50: 50, \mathrm{v} / \mathrm{v}) \mathrm{with}$ isocratic mode. The

detection wavelength was $245 \mathrm{~nm}$

\section{Tuning of endothelial monolayer permeability by agonistic micelles}

Para-endothelial TJs play a critical role in maintaining brain homeostasis and regulating $\mathrm{BBB}$ permeability. The TJs are comprised of transmembrane $\mathrm{TJ}$ proteins and intracellular scaffolding proteins that anchor the TJ proteins to an actin cytoskeleton. The dynamics of occludin (representing TJ proteins), zonula occluden-1 (ZO-1) (representing scaffolding proteins) and actin stress fiber (ASF) (representing actin cytoskeleton) were investigated after the treatment of normoxia and oxygen-glucose deprivation (OGD) $\left(1 \% \mathrm{O}_{2}\right.$ for $2 \mathrm{~h}$ ). This was followed by reoxygenation $\left(21 \% \mathrm{O}_{2}\right.$ for $\left.2 \mathrm{~h}\right)$, or OGD $(2 \mathrm{~h})$ followed by reoxygenation in the presence of EDV-AM (2 h). Immuno-fluorescence images of the fully confluent endothelial bEnd.3 cell monolayer showed that both occludin and ZO-1 distributed as sharp paracellular alignments under normal conditions (Figure 3A). In contrast, OGD/reoxygenation not only led to the disruption and diffusion of both paracellular ZO-1 and occludin profiles but also triggered their translocation from the cell periphery to the perinuclear area. The ASF were observed as thin and parallel fibers under normal conditions. However, OGD/reoxygenation dramatically increased the thickness and length of ASF by forming spindle-like bundles that orientated predominately along the longitudinal axis of the cells. The compromised TJs after hypoxic treatment could be explained by the up-regulated inflammatory factors, proteolytic enzymes, and Rho-kinases that further modulate stress fiber formation [30], myosin light chain phosphorylation, and nitric oxide bioavailability [31]. The expression levels of ZO-1, Occludin, and ASF were barely changed before and after EDV-PM treatment (Figure S2), verifying that the agonistic micelle-induced $\mathrm{A}_{2 \mathrm{~A}} \mathrm{R}$ signaling underlies the TJ compromise. More pronounced reduction of the paracellular $\mathrm{ZO}-1$ and occludin profiles were observed after treatment of OGD/reoxygenation/EDV-AM. Also, the reorganization of ASF indicating cell contraction and TJ dysfunction were more evident. These results showed that the agonistic micelle could enhance BBB permeability in brain ischemia by amplifying the TJ-associated protein attenuation and ASF contraction.

The capability of EDV-AM to tune the permeability of an in vitro BBB model upon OGD/reoxygenation $\left(1 \% \mathrm{O}_{2}\right.$ for 2 hours followed $21 \%$ $\mathrm{O}_{2}$ for $24 \mathrm{~h}$ ) treatment was examined (Figure 3B). PBS, EDV-AM or EDV-PM was added to a confluent endothelial bEnd.3 cell monolayer at the end of OGD treatment and the time-dependent transendothelial cell electrical resistance (TEER) values were measured during the reoxygenation. While the TEER value remained around $118.7 \Omega \bullet \mathrm{cm}^{2}$ under the normoxic condition, it was measured to be $83.1 \Omega \Omega^{\circ} \mathrm{cm}^{2}$ immediately after OGD treatment. The TEER value decreased continuously after addition of PBS or EDV-PM $(10 \mu \mathrm{M})$ and an additional 30\% TEER reduction was recorded after reoxygenation for $3 \mathrm{~h}$ which could be explained by the ongoing $\mathrm{TJ}$ disruption during the reoxygenation stage. Notably, the TEER value gradually recovered to $81.6 \pm 1.50 \%$ of its initial value $8 \mathrm{~h}$ post reoxygenation implying the self-restoration of the TJ integrity. Treatment with EDV-AM $(10 \mu \mathrm{M})$ led to a rapid TEER reduction with a maximal value of $62.5 \pm 0.95 \%$ in the first hour, confirming the cell monolayer permeability enhancement after the agonistic micelles treatment. EDV-AM increased the cell monolayer permeability in a concentration-dependent manner. The TEER maximally decreased $67.1 \pm 1.11 \%, 62.5 \pm 0.95 \%, 55.8 \pm$ $9.06 \%$ and $46.6 \pm 5.61 \%$ after $1 \mathrm{~h}$ treatment with 40,10 , 1.0 and $0.1 \mu \mathrm{M}$ EDV-AM, respectively (Figure 3C, Figure S3). Notably, the TJ opening time-windows (> $40 \%$ TEER reduction) correlated with the EDV-AM concentration and were measured at $1,2,2$, and $4 \mathrm{~h}$ after treatment of $0.1,1.0,10$, and $40 \mu \mathrm{M}$ EDV-AM, respectively. It is also of note that the TEER value recovered above $82.5 \%$ of its initial value $12 \mathrm{~h}$ post EDV-AM treatment regardless of its concentration. The recovered TEER value indicated the minimized TJ injuries induced by the agonistic micelle-mediated $\mathrm{A}_{2 \mathrm{~A}} \mathrm{R}$ signaling. Above studies confirmed that EDV-AM could temporarily enhance cell monolayer permeability after the OGD/reoxygenation treatment. 

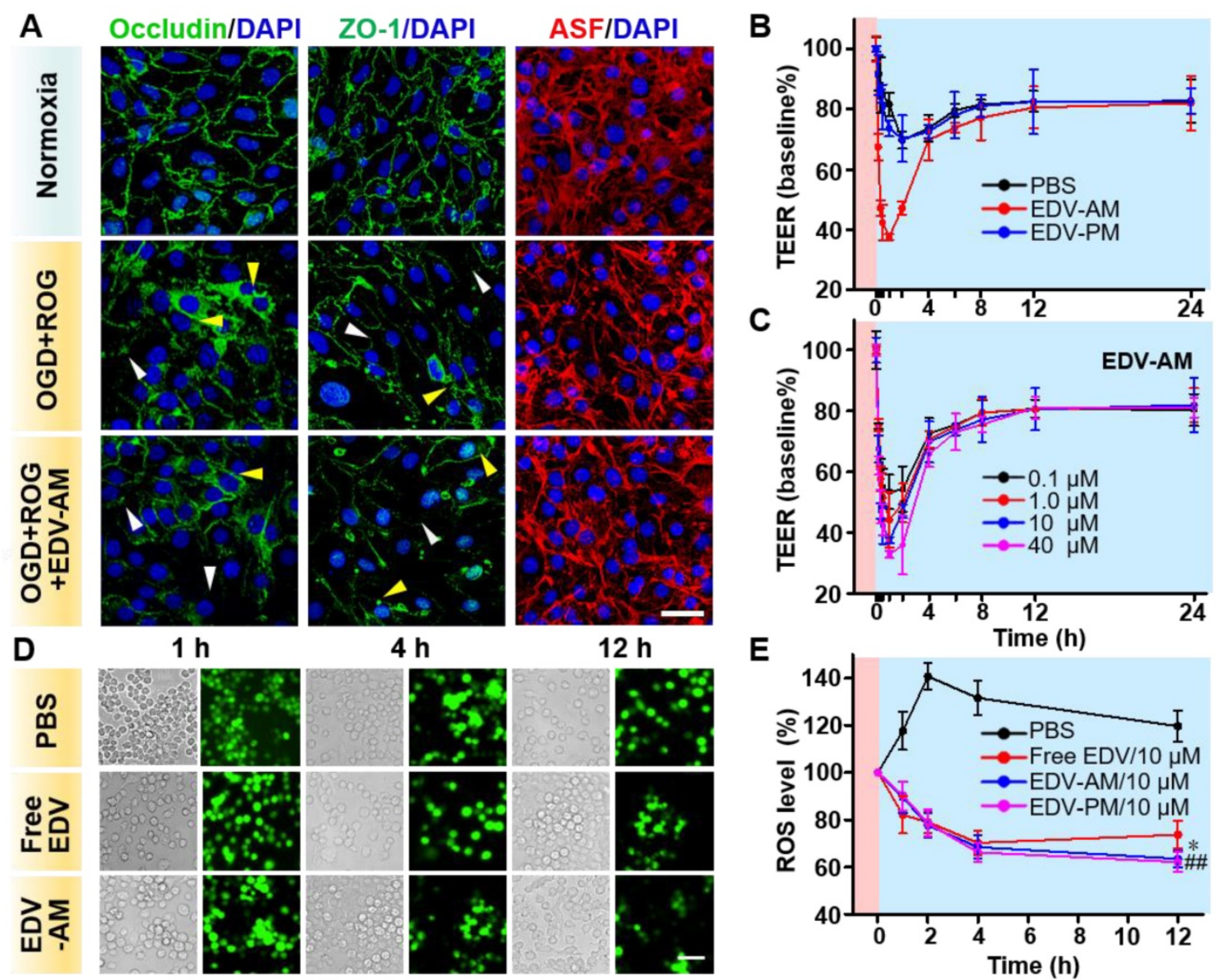

Figure 3. Agonistic micelles tune endothelial monolayer permeability and eradicate OGD-induced intracellular ROS. (A) Fluorescence microscopic images of TJ-associated proteins ZO-1, occluding, and actinomyosin stress fibers (ASF) in endothelial bEnd.3 monolayer after the treatment of normoxia, OGD ( $2 \mathrm{~h}$ ) followed by ROG (2 $\mathrm{h})$, or OGD ( $2 \mathrm{~h}$ ) followed by ROG $(2 \mathrm{~h})$ plus EDV-AM $(10 \mu \mathrm{M})$. ASF stained with phalloidin is displayed in red, immunofluorescence of ZO- 1 and occludin is in green, nuclei stained with DAPI are in blue. White and yellow arrow heads point to the disrupted paracellular T] proteins and the translocated TJ proteins in the perinuclear area. Scale bar, $50 \mu \mathrm{m}$. (B) Time-dependent TEERs of OGD-treated bEnd. 3 cell monolayer after addition of PBS, EDV-AM or EDV-PM (10 $\mu M)$. Magenta and cyan backgrounds indicate OGD and reoxygenation stages, respectively. (C) TEERs of OGD-treated bEnd.3 monolayer as functions of EDV-AM concentrations. (D) White light and fluorescence images of OGD-treated live RAW264.7 microphage cells at selected time-points post addition of PBS, free EDV, or EDV-AM. (E) Normalized intracellular ROS level as a function of incubation time after addition of EDV-AM, free EDV, or PBS. $* P<0.05$, EDV-AM vs. free EDV group; \# $P<0.01$, EDV-AM vs. PBS group, OGD: oxygen and glucose deprivation. ROG: reoxygenation.

\section{Efficient eradication of intracellular ROS by agonistic micelles}

Over-production of ROS in live RAW264.7 microphages was evident after a 2-h OGD treatment (Figure 3D). At the end of OGD treatment, PBS, EDV-AM, EDV-PM, or free EDV was added to the cell cultures followed by reoxygenation. While the intracellular fluorescence intensity of the ROS probe increased by $40.6 \pm 5.71 \%$ at $2 \mathrm{~h}$ post reoxygenation, an obvious signal reduction was observed after the treatment of free EDV, EDV-PM, or EDV-AM (10 $\mu \mathrm{M})$. Even though free EDV showed higher ROS elimination velocities than that of EDV-AM in the first $2 \mathrm{~h}$ reoxygenation, the agonistic micelle showed a more persistent ROS quenching effect with significantly lower ROS level than that of the free EDV from 4 to $12 \mathrm{~h}$ (Figure 3E). The persistent intracellular ROS elimination implied the efficient internalization and sustainable EDV release of EDV-AM in the macrophages (Figure S4).

\section{High uptake in brain ischemia by agonistic micelles}

In vivo near-infrared (NIR) fluorescent imaging showed higher fluorescence signal of EDV-AM than that of EDV-PM on the ischemic mouse head after intravenous (i.v.) injection (Figure 4A). The EDV-AM signal in brain areas increased steadily and reached its maximum $\left(12.1 \pm 0.99 \times 10^{8} \mathrm{p} / \mathrm{s} / \mathrm{cm}^{2} / \mathrm{Sr}\right)$ at $2 \mathrm{~h}$ post-injection (PI), whereas the EDV-PM signal reached its highest value $\left(8.6 \pm 0.33 \times 10^{8} \mathrm{p} / \mathrm{s} / \mathrm{cm}^{2} / \mathrm{Sr}\right)$ at $1.0 \mathrm{~h} \mathrm{PI}$ and then decreased consistently (Figure 4B). Ex vivo NIR fluorescence images verified the higher fluorescence signals of EDV-AM than that of EDV-PM in brain ischemia. The fluorescence intensity in the ischemic hemisphere was determined to be $4.6 \times 10^{9} \mathrm{p} / \mathrm{s} / \mathrm{cm}^{2} / \mathrm{Sr} 24 \mathrm{~h}$ PI of EDV-AM which is 2.1 times higher than that of EDV-PM (Figure 4C). EDV concentrations in the homogenized extraction of 
ischemic brain hemisphere were measured by analytic HPLC at $24 \mathrm{~h}$ PI with respect to free EDV, EDV-AM or EDV-PM with the same EDV dose $(0.078 \mathrm{mg}$ $\mathrm{EDV} /$ mouse) (Figure 4D). The highest EDV concentration was determined as $0.0583 \pm 0.0011 \mu \mathrm{g} / \mathrm{g}$ after injection of EDV-AM and was 2.0 and 7.7-fold higher than the values after administration of EDV-PM $(0.029 \pm 0.019 \mu \mathrm{g} / \mathrm{g})$ or free EDV $(0.0075 \pm$ $0.0031 \mu \mathrm{g} / \mathrm{g}$ ) (Figure 4E).

\section{Rescue of ischemic brain tissue by agonistic micelles}

The ischemic stroke mouse models were randomly divided into four groups that were injected with PBS, free EDV, EDV-AM or EDV-PM at 1, 4 and 7 days post model establishment (Figure 5A). The neuroprotective efficacy was non-invasively evaluated by T2-weighted (T2W) MRI that delineated ischemic regions with hyper-intensive signals. EDV-AM showed a $75.2 \pm 1.8 \%$ ischemic volume reduction at 7 days post its treatment $(\mathrm{n}=5$ animal models). In contrast, $25.6 \pm 4.5 \%, 36.5 \pm 13.1 \%$ and 48.8 $\pm 8.3 \%$ ischemic volume reductions were recorded after treatment of PBS, EDV-PM or free EDV, respectively. At 14 days post-treatment, only residual ischemia (13.0 $\pm 3.6 \%$ of original ischemic volume) remained in the EDV-AM treated group, whereas 67.1 $\pm 3.1 \%, 55.0 \pm 3.1 \%$ and $51.2 \pm 5.7 \%$ original ischemic volumes were still present after the treatment with
PBS, EDV-PM or free EDV, respectively, $(n=5)$. It is of note that while the infarct volume decreased remarkably from $24.9 \pm 1.8 \%$ at day 7 to $13.9 \pm 3.6 \%$ at day 14 post EDV-AM treatment, the ischemic area was barely changed in the groups treated with free EDV or EDV-PM after excluding the spontaneous rehabilitation (Figure 5B). The pathological BBB permeability enhancement in brain ischemia was reported to occur as a biphasic response [23]. The first phase, occurring in 2-8 hours after stroke onset, was induced by the ROS-triggered TJ protein degradation. The second phase occurred 18-96 hours later and was a result of vasogenic edema and angiogenesis. Notably, while EDV-AM, EDV-PM, and free EDV all were remarkably reduced ischemic volume in the first 4 days after treatment, obvious neuroprotective effects from day 7 to day 14 was only observed after EDV-AM treatment. The positive therapeutic effect in the first 4 days could be partially explained by the enhanced EDV brain uptake because of the pathologically compromised BBB. The recovered BBB integrity from day 7 to day 14 prevents the cerebral uptake of EDV and EDV-PM which could explain why only EDV-AM with BBB permeability showed the neuroprotective effect. Our studies showed the feasibility to extend the therapeutic time-window of ischemic stroke via the agonistic micelles-mediated brain delivery of neuroprotectants.
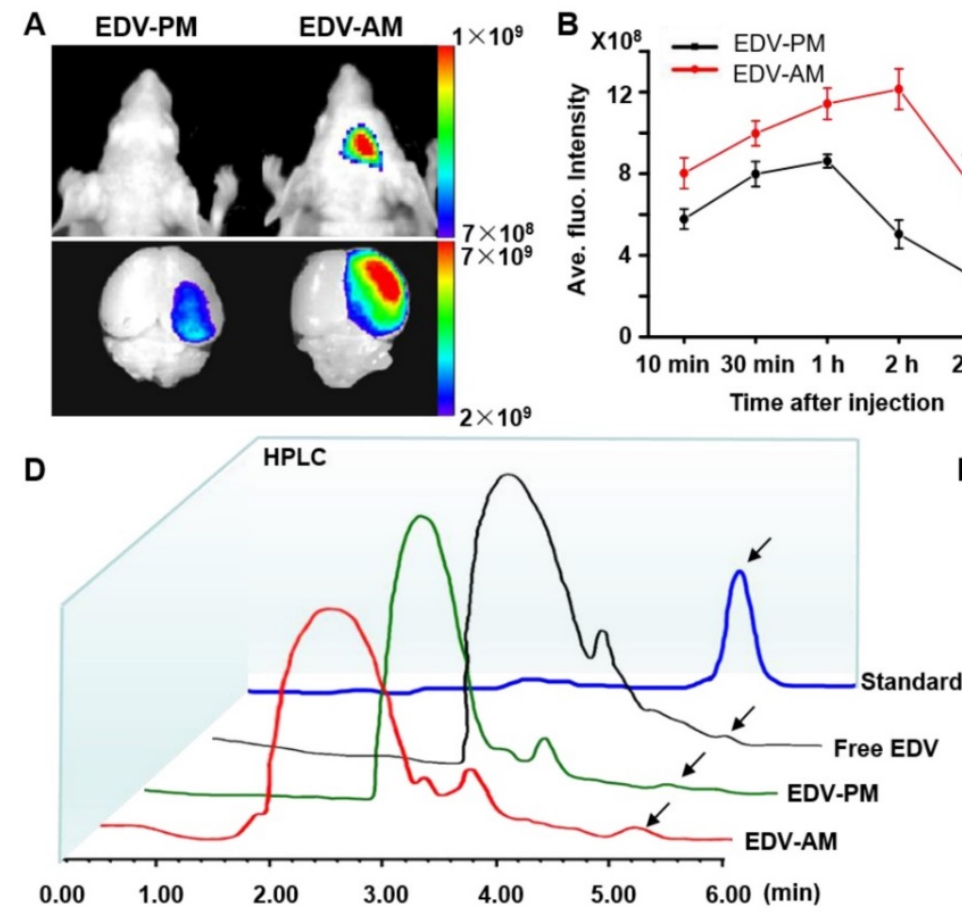

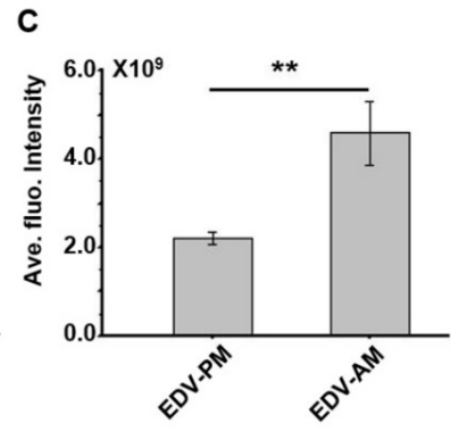

E

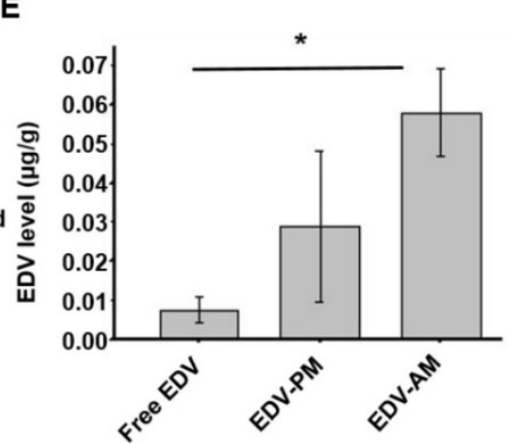

Figure 4. Agonistic micelles increase EDV uptake in brain ischemia. (A) NIR fluorescence images of the mouse brain at $24 \mathrm{~h} P I$ of EDV-AM or EDV-PM (0.078 mg $\mathrm{EDV} /$ mouse) via i.v.. The treatments were conducted at $6 \mathrm{~h}$ post-stroke model establishment. Scale unit: $\mathrm{p} / \mathrm{sec} / \mathrm{cm}^{2} / \mathrm{Sr}$. (B) In vivo time-dependent normalized NIR fluorescence intensities in mouse brain area after injection of EDV-AM or EDV-PM (mean \pm SD, $n=5$ ). $* * P<0.01$, EDV-AM vs. EDV-PM group. (C) Ex vivo NIR fluorescence intensities in the excised brain ischemia at $24 \mathrm{~h}$ PI of EDV-AM or EDV-PM $(n=5)$. (D) HPLC chromatography of homogenized extractions of the ipsilateral hemisphere at $24 \mathrm{~h}$ PI of free EDV, EDV-PM, or AM-EDV. Arrows point to the eluting peak of EDV. (E) Quantification of EDV concentration in the ipsilateral hemisphere at $24 \mathrm{~h}$ PI free EDV, EDV-PM or EDV-AM. $* P<0.05$ EDV-AM vs. free EDV group. PI: post-injection. 
A

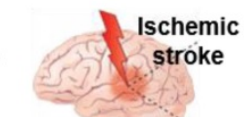

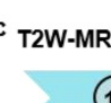

(1d fo T2W-M r

(4d)

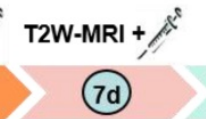

T2W-MRI + DTI-MRI

(14d)

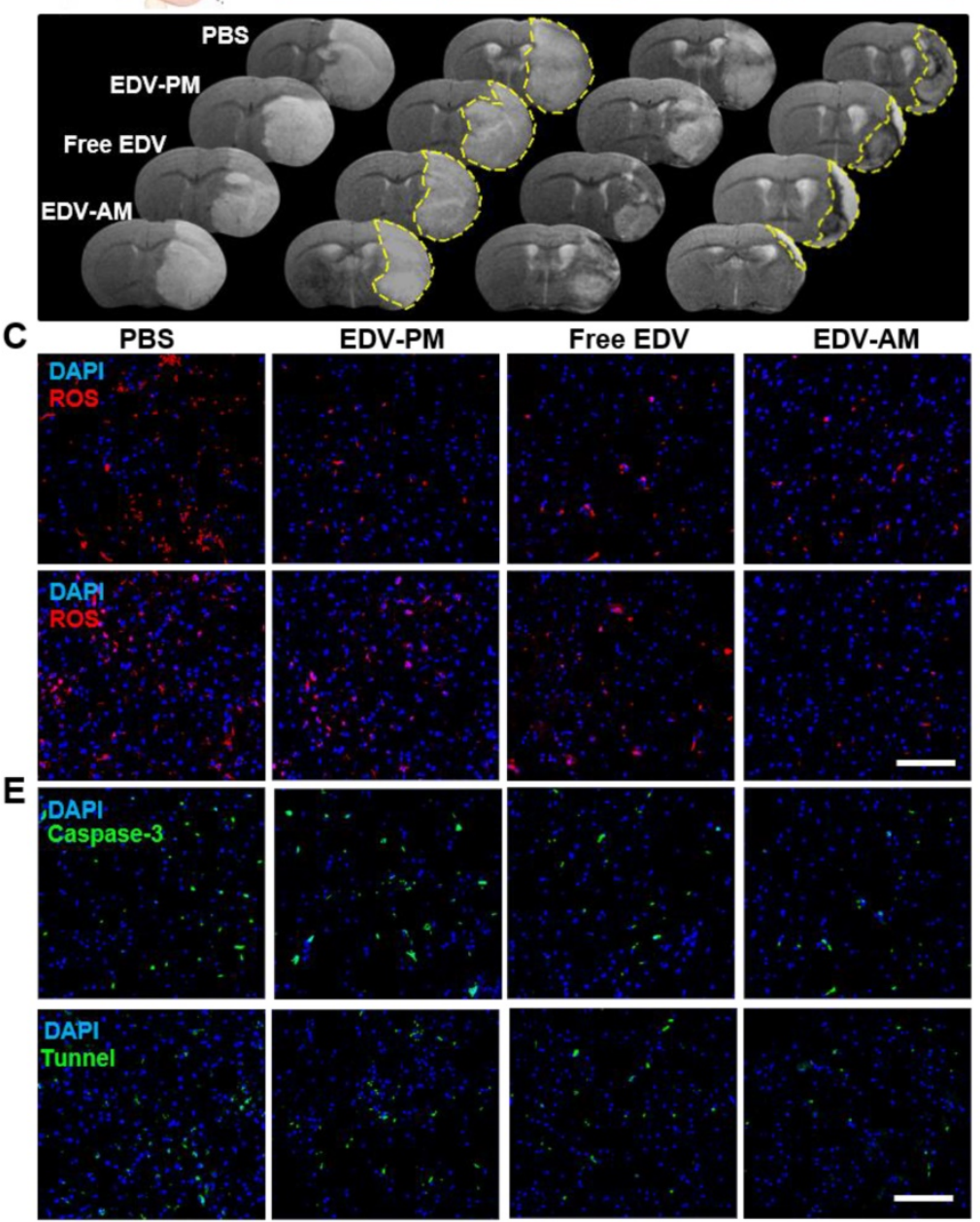

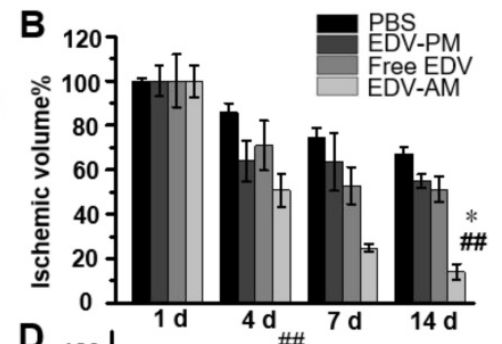
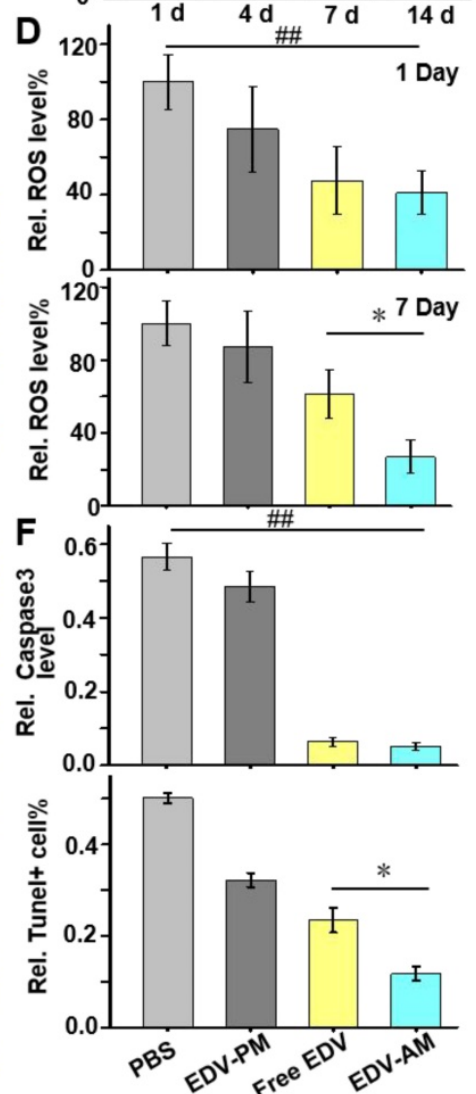

Figure 5. Agonistic micelles salvage ischemic brain tissue by eradicating ROS. (A) Representative in vivo T2W-MRI of the ischemic mouse brain at 1,4 , 7, and 14 day PI of PBS, EDV-AM, or EDV-PM with the same EDV dose $(0.078 \mathrm{mg} / \mathrm{mouse})$ via i.v.. Above treatments were conducted at $6 \mathrm{~h}$ post-stroke model establishment. The ischemic regions at 4 and 14 days post treatment were marked by yellow dashes. (B) The ratio between ischemic and whole brain volume at selected times post treatments ( $\mathrm{n}=4$ or 5 mice per group). \# $P<0.01$ vs. PBS group, $* P<0.05$ vs. free EDV group. (C) Fluorescence microscopic images displaying ROS levels in ischemic brain striatum at $24 \mathrm{~h}$ and 7 days post treatments. Intracerebral ROS were visualized by the i.v. injected dihydroethidium (HEt) that was oxidized by ROS to ethidium (Et) with red color fluorescence. (D) Quantification of ROS levels in ischemic brain striatum at $24 \mathrm{~h}$ and 7 days post treatments $(\mathrm{n}=12$ slices from 4 mouse models). (E) Fluorescence microscopic images of ischemic brain striatum stained by Tunnel kit or immunostained by caspase- 3 antibody at 14 days post treatments. Tunnel + or caspase- 3 immunofluorescence was displayed in green and nuclei were in blue ( $n=12$ slices from 3 animal models). $(F)$ Tunnel + and active caspase- 3 fluorescence intensities in the ipsilesional striatum post treatments ( $n=12$ slices from 4 mouse models). \#\# $P<0.01, * P<0.05$. Scale bar, $100 \mu \mathrm{m}$.

The antioxidant effect of the agonistic micelles in brain ischemia was investigated by dihydroethidium (HEt) assay. The BBB permeable compound HEt could be oxidized by intracerebral ROS to ethidium (Et) that intercalates with DNA to generate a bright red color fluorescence [32]. The Et fluorescence intensity in the ipsilateral hemisphere was significantly higher than that in the contralateral hemisphere at both $24 \mathrm{~h}$ and 7 days post model establishment indicating consistent over-production of ROS in the brain ischemia (Figure S5). Et signal reductions in brain ischemia were obvious after treatment of free EDV, EDV-PM or EDV-AM, which indicates the down-regulation of cerebral ROS level
(Figure 5C). Significantly, while free EDV and EDV-AM groups offered similar Et signal reductions in the ipsilateral striatum at $24 \mathrm{~h}$, EDV-AM group demonstrated the lowest Et signal that was 2.3, 3.2 and 3.7 times lower than that of free EDV, EDV-PM, and PBS groups, respectively, at 7 day post-treatment (Figure 5D). Caspase-3, an executioner caspase inducing internucleosomal chromatin condensation and DNA fragmentation is widely used as an early marker of apoptosis during acute cerebral ischemia $[33,34]$. Immunofluorescent staining showed that the average caspase-3 expression level in the ipsilesional striatum at 14-day post EDV-AM treatment was 10.9, 9.3, and 1.2 times lower than that treated with PBS, 
EDV-PM, and free EDV, respectively (Figure 5E-F). Neuronal DNA fragmentation as a marker of late-apoptosis was quantified via terminal-deoxynucleotidyl-transferase mediated dUTP nick end labeling (TUNEL) staining at 14 days post treatments. The TUNEL-positive cells in the ipsilesional striatum after EDV-AM treatment were determined to be $11.7 \pm 1.5 \%$ that was $4.3,2.7$, and 2.0-fold lower than that after treatment of PBS, EDV-PM, and free EDV, respectively (Figure 5E-F).

Safely tuning BBB permeability in brain ischemia is the key to the success of agonistic micelles-mediated neuroprotective therapy. Recently, we systematically investigated the security of a dendrimer-based $\mathrm{A}_{2 \mathrm{~A}} \mathrm{R}$ nanoagonist in normal mice [35]. Paracellular TJ opening with an average width of $25 \mathrm{~nm}$ was observed in TEM images at $2 \mathrm{~h}$ post injection of the nanoagonist, which allows the passage of most small molecular compounds, macromolecular therapeutics, and polymetric drug delivery vectors. However, the blood-derived cells such as erythrocytes $(\sim 7-8 \mu \mathrm{m})$ and leucocytes $(\sim 7-20 \mu \mathrm{m})$, which reduce the blood cells extravasation-induced immunogenicity and neurologic deterioration, could not pass through the small TJ openings. Importantly, timely TJ restoration was observed at $4 \mathrm{~h}$ post nanoagonist administration minimizing the $\mathrm{TJ}$ breakdown associated vasogenic edema and neuronal apoptosis [35]. The minimized side effects of the nanoagonist can be explained by the limited compromise and rapid restoration of the $\mathrm{TJ}$ integrity after $\mathrm{A}_{2 \mathrm{~A}} \mathrm{R}$-mediated endogenous intracellular signaling. Compared to the dendrimer-based nanoagonist, agonistic micelles should be even safer due to better biocompatibility, full degradability, and neutral surface charge that minimize non-specific accumulation in normal tissues. In fact, the minimized apoptosis and edema in ischemic brain tissue after multiple injections of the agonistic micelles verified the low side-effects of $\mathrm{A}_{2 \mathrm{~A}} \mathrm{R}$-mediated $\mathrm{TJ}$ opening strategy.

Compared to the widely used filament-mediated MCAo models, photochemically induced permanent MCAo model used in this study showed several advantages including high success rate $>95 \%$ in our lab), controllable infract volume, and long-term model survival (100\% after one month), which were particularly important to assess therapeutic effectiveness. Photothrombotic ischemic stroke models have been widely used in accessing post-stroke animal behaviors [36], cerebrovascular inflammation following thrombolytic reperfusion and therapeutic response after endothelial progenitor cell (EPC) transplantation [37, 38]. Even though endovascular thrombus/clot blocked the blood flow in the laser irradiated artery, the micelles still could be delivered into brain ischemia via collateral circulation in the subsidiary network of vascular channels supplementing cerebral blood flow when principal conduits fail [39]. In this study, the agonistic micelles could reach brain ischemia via collateral circulation and enter brain parenchyma by actively enhancing BBB permeability.

\section{Improvement in axonal remodeling and reduction in behavioral deficits by agonist micelles}

Compared to T2W-MRI, diffusion tensor imaging (DTI) elucidates distinct pathologic changes such as structural abnormalities of white matter fibers after cerebral ischemia. In vivo DTI quantitatively outlined the directions as well as spatial distribution of white matter fibers in the ipsilesional and contralateral inner capsule (IC) where the ischemia-sensitive motor and sensory fibers are abundant (Figure 6A). The fiber track thickness and connectivity in the ipsilesional IC increased remarkably after EDV-AM treatment indicating the improved axonal remodeling in brain ischemia. Cross-sectional DTI further quantitatively evaluated the fiber track integrity in the ipsilesional IC by determining fractional anisotropy (FA) that measures the directional degree of water diffusion (Figure 6B). The FA value in ipsilesional IC after EDV-AM treatment was determined to be 0.7814 days PI which was significantly higher than that after treatment with EDV $(0.54 \pm 0.13)$, EDV-PM $(0.55 \pm 0.062)$ or PBS $(0.34$ \pm 0.13 ). Limited by cell membranes and myelin fibers, the water diffusion coefficient in the brain is often anisotropic and direction dependent. In normal white matter, FA value is high due to the thick fiber bundles running in a uniform direction with preserved water diffusion along the axons. In ischemic regions, FA values are low because of ischemic injury-associated fiber integrity loss and edema-induced tract disorganization. The highest FA value elevation after EDV-AM intervention implied its best neuroprotective effect. EDV-AM markedly prolonged the survival of the ischemic stroke mouse models (Figure 6C). The median survival of the models treated with the EDV-AM was determined to be $42.9 \%$ at day 14 and was significantly higher than those treated with PBS (14.3\%), EDV (28.6\%), and EDV-PM (28.6\%). Figure 6D-E present the time-dependent profiles of functional recovery after different treatments. Animal models treated with EDV-AM showed the largest reduction of the modified neurological severity score (mNSS) that quantitatively determined the neurological functional deficits with respect to motor, sensory, reflex and 
balance criteria (normal score: 0, maximal deficit score: 18). The mNSS value at 14 days after treatment of EDV-AM was determined to be 1.25 that was significantly lower than the stroke models treated with PBS (4.6 \pm 1.15$)$, EDV $(4.3 \pm 0.58)$ and EDV-PM $(3.5 \pm 0.57)$ (Figure 6D). Foot-fault test assessing the locomotor activity showed the lowest value in the models after EDV-AM treatment. The value at 14 days PI was measured as $13.0 \%$ that was significantly lower than that treated with PBS $(16.6 \% \pm 1.55 \%)$, $\operatorname{EDV}(17.1 \% \pm 1.77 \%)$, or EDV-PM $(18.0 \% \pm 0.85 \%)$ (Figure 6E).

\section{Conclusion}

Herein, we developed a pluripotent agonist micelle EDV-AM to rescue brain ischemic tissues by specifically up-regulating cerebral uptake of ROS scavenger EDV. EDV-AM specifically up-regulates BBB permeability, delivered EDV with high payload into brain ischemia and efficiently eradicates intracerebral ROS. Experimental results showed the rapid infarct volume reduction, prolonged survival, improved axonal remodeling, and reduced behavioral deficits in animal models following treatment with EDV-AM. Significantly, remarkable neuroprotective effects were observed even though the agonistic micelles were injected as long as 4 days after the onset of the infarct attack. In summary, EDV-AM shows a promising way to improve neuroprotective effects by sustainably eradicating cerebral ROS. Especially, EDV-AM treatment would be beneficial to the majority of ischemic stroke patients who miss the narrow time window for the thrombolytic therapy.
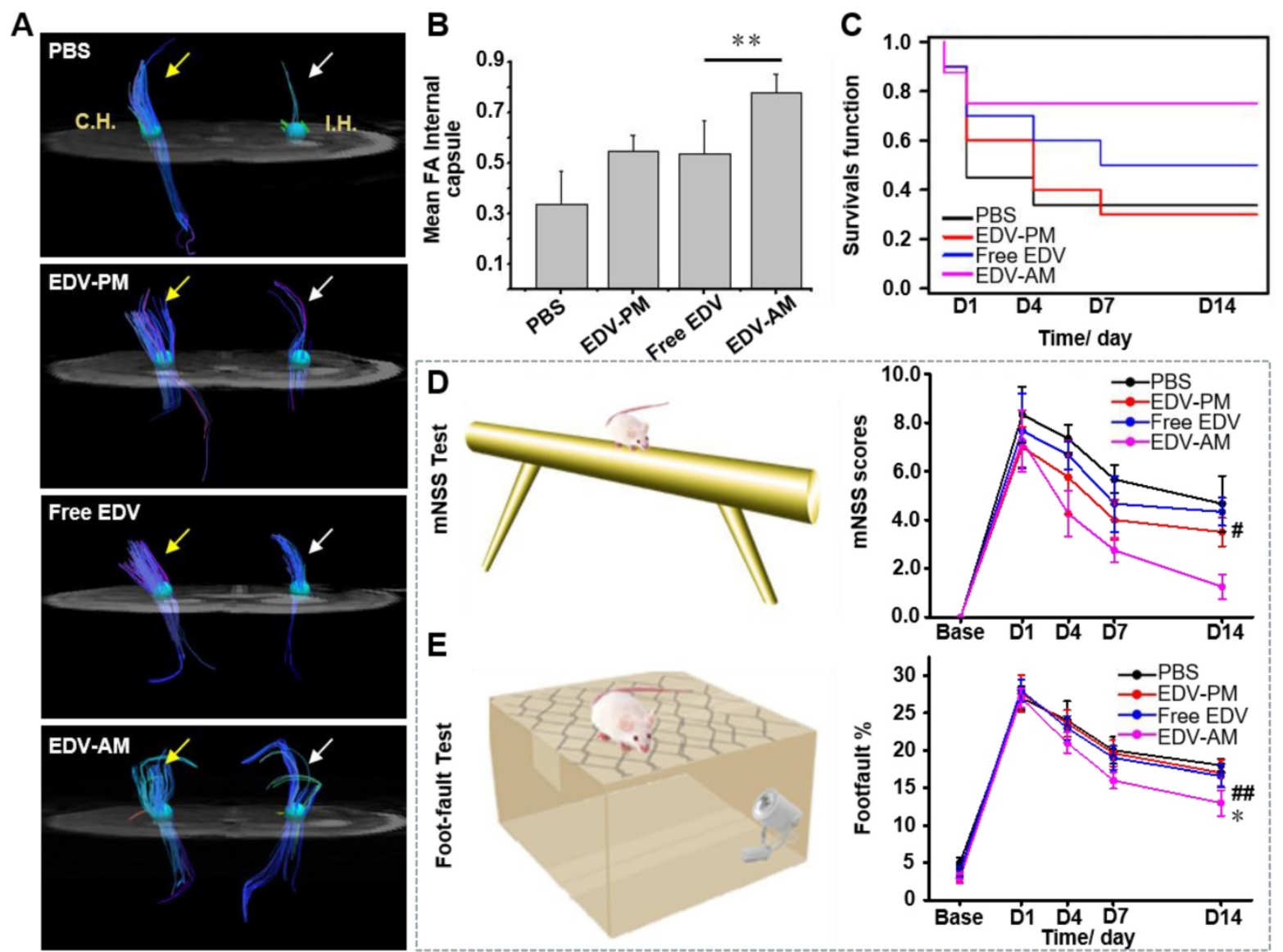

Figure 6. Agonistic micelles improve axonal remodeling and reduce behavioral deficits. (A) In vivo DTI demonstrated fiber tract integrity and orientation in the ipsilesional and contralateral inner capsule at 14 days post injection of PBS, free EDV, EDV-AM, or EDV-PM with the same EDV dose (0.078 mg/mouse). White and yellow arrows point to fiber tract in ipsilateral and contralateral IC, respectively. (B) Fractional anisotropy $(F A)$ values determined in the ipsilesional IC at 14 days post treatment ( $\mathrm{n}=$ $4-5$, ** $P<0.01$ vs. free EDV group). (C) Kaplan-Meier survival curves of the ischemic stroke models at 1,4 , and 7 days post treatments indicated in Figure $5 A$. Behavioral deficits in the animal models were evaluated using modified neurological severity scores (D) and foot-fault test (E) before and at $1,4,7$, and 14 days ( $n=5-6$ per group). \# $P<0.05$, \#\# $P<0.01$ vs. PBS group, $* P<0.05$ vs. free EDV group. $\mathrm{CH}$ : contralateral hemisphere, IH: ipsilateral hemisphere, IC: inner capsule. 


\section{Materials \& Methods}

\section{Materials}

All chemical reagents were obtained from Aladdin Reagent (Shanghai, China) unless otherwise specified. CGS21680 was purchased from Chengdu Novi Biotech. (Chengdu, China). Maleimide-PEG (2.0 $\mathrm{kDa})$-PLA $(2.0 \mathrm{kDa})$ and $\mathrm{CH}_{3} \mathrm{O}-\mathrm{PEG}(2.0 \mathrm{kDa})$-PLA $(2.0 \mathrm{kDa})$ were acquired from JenKem Tech. (Beijing, China). EDV was purchased from Dalian MeiLun Biotech. (Dalian, China). Cell culture media, fetal bovine serum (FBS), trypsin and penicillin and streptomycin (PS) were from Gibco (Waltham, USA). Amicon ultra-15 centrifugal filter tubes $(3,000$ and 10,000 MW cutoff) were from Millipore (Bedford, USA). Rabbit anti-mouse caspase- $3, \mathrm{~A}_{2 \mathrm{~A}} \mathrm{R}$, occludin and ZO-1 primary antibodies were purchased from Abcam (Cambridge, UK). Alexa Fluo488 labeled donkey anti-rabbit secondary antibody was obtained from Cell Signaling Tech. (Danvers, USA). Goat anti-mouse CD31 primary antibody and Fluor555-labeled donkey anti-goat secondary antibody were provided by Santa Cruze (Dallas, USA). TUNEL apoptosis assay kit was purchased from KeyGEN Biotech. (Nanjing, China). ROS Assay Kit was acquired from Beyotime Biotech. (Nantong, China).

\section{Synthesis}

Briefly, CGS-21680 (5.4 mg, $10 \mu \mathrm{mol})$, HATU (7.6 $\mathrm{mg}, 20 \mu \mathrm{mol})$ and triethylamine $(2.0 \mathrm{mg}, 20 \mu \mathrm{mol})$ mixed in $1.0 \mathrm{~mL}$ anhydrous DMF were stirred at room temperature (r.t.) for $30 \mathrm{~min}$. Then 2-aminoethanethiol $(1.2 \mathrm{mg}, 15 \mu \mathrm{mol})$ in $150 \mu \mathrm{L}$ DMF was added. After stirring for $24 \mathrm{~h}$ at r.t., the product was purified using silica gel column chromatography to yield CGS-HS (1). The reaction between Mal-PEG-PLA (10 mg, 2.5 $\mu \mathrm{mol})$ and compound $1(1.4 \mathrm{mg}, 2.5 \mu \mathrm{mol})$ in PBS ( $\mathrm{pH}$ 7.4) at r.t. gave rise to CGS-PEG-PLA (2). IRB-HS was obtained by the reaction of IRB-COOH $(8.6 \mathrm{mg}, 10$ $\mu \mathrm{mol})$ with 2-aminoethanethiol $(1.2 \mathrm{mg}, 15 \mu \mathrm{mol})(3)$. Compound $3(9.2 \mathrm{mg}, 10 \mu \mathrm{mol})$ treated with Mal-PEG-PLA (40 mg, $10 \mu \mathrm{mol})$ in PBS resulted in IRB-PEG-PLA (4). Agonistic micelle EDV-AM and control micelle EDV-PM were synthesized through an emulsion/solvent evaporation strategy [40]. MeO-PEG-PLA (33.4 mg, $8.4 \mu \mathrm{mol})$, IRB-PEG-PLA (0.7 mg, $0.2 \mu \mathrm{mol})$, CGS-PEG-PLA $(0.1 \mathrm{mg}, 0.03 \mu \mathrm{mol})$ and $\operatorname{EDV}(1.2 \mathrm{mg}, 6.7 \mu \mathrm{mol})$ dissolved in $1.0 \mathrm{~mL}$ acetonitrile were sonicated on ice for 15 seconds. Evaporating the solvent at $40^{\circ} \mathrm{C}$ and re-suspending the mixture in $3.0 \mathrm{~mL}$ PBS yielded EDV-AM. EDV-PM was synthesized using a similar method but without CGS-PEG-PLA in the starting materials.

\section{Cell culture}

Immortalized mouse brain capillary endothelial bEnd.3 cell line and murine macrophage RAW264.7 cell line were purchased from American Type Culture Collection (ATCC) (Manassas, USA) and cultured in Dulbecco's modified Eagle's medium (DMEM) supplemented with $10 \%$ FBS and $1 \%$ penicillin-streptomycin (PS). Cells were maintained in a humidified cell culture incubator at $37^{\circ} \mathrm{C}$ with $5 \%$ carbon dioxide $\left(\mathrm{CO}_{2}\right)$.

\section{In vitro oxygen-glucose deprivation (OGD)/reoxygenation studies}

In vitro cell culture studies with OGD and reoxygenation treatments were performed following the method described previously [41]. Briefly, the cells with a confluence of $80-100 \%$ were cultured in a deoxygenated, glucose-free DMEM medium and then placed in a humidified incubator supplied with an anaerobic gas mixture $\left(94 \% \mathrm{~N}_{2}, 1 \% \mathrm{O}_{2}\right.$ and $\left.5 \% \mathrm{CO}_{2}\right)$ at $37^{\circ} \mathrm{C}$. After $2 \mathrm{~h}$ incubation, the cells were removed from the anaerobic incubator and the OGD media (glucose-free DMEM medium, 1\% penicillin-streptomycin) was replaced followed by reoxygenation by culturing in a regular incubator $\left(21 \% \mathrm{O}_{2}\right.$ and $\left.5 \% \mathrm{CO}_{2}\right)$.

\section{Measuring intracellular ROS level after OGD treatment}

Intracellular ROS levels under normoxia and hypoxia were quantitated by using $\mathrm{DCFH}_{2}-\mathrm{DA}$ as a ROS reporter [42]. Briefly, RAW264.7 cells at a density of $2 \times 10^{5}$ cells/well in a 24-well plate were cultured in OGD for $2 \mathrm{~h}$. At the end of treatment, PBS, free EDV or EDV-AM with a final EDV concentration of 1.0, 10, and $100 \mu \mathrm{M}$ were added immediately. The cells were continuously cultured under the reoxygenative condition for 1,2 , and $12 \mathrm{~h} .10 \mu \mathrm{M} \mathrm{DCFH}_{2}$-DA was added at the end of treatment and the cells were incubated at $37^{\circ} \mathrm{C}$ for another $30 \mathrm{~min}$. The ROS levels were measured by quantifying the intracellular fluorescence intensities [43]. The $\mathrm{DCFH}_{2}$ fluorescence images were collected under a fluorescence microscope with a band-pass excitation filter $488 \mathrm{~nm}$ and emission filter at $525 \mathrm{~nm}$.

\section{Establishment of in vitro BBB model}

Endothelial bEnd.3 cells were seeded on the luminal side of the transwell filter $(0.4 \mathrm{~mm}$ pore size $)$ insert with a density of $8.0 \times 10^{4}$ cells/filter $\left(1.12 \mathrm{~cm}^{2}\right.$ surface area). The cells were cultured in DMEM supplemented with $10 \%$ FBS and $1 \%$ PS $(0.6 \mathrm{~mL}$ and $1.2 \mathrm{~mL}$ in the luminal and albumin compartments respectively). Trans-endothelial cell electrical resistance (TEER) values were measured daily to 
evaluate the cell monolayer permeability until the TEER value attained $190-210 \Omega \cdot \mathrm{cm}^{2}$. After culturing under OGD condition for $2 \mathrm{~h}$, PBS, free EDV, EDV-PM, or EDV-AM was added and the cell monolayers were treated under the reoxygenative condition for another $24 \mathrm{~h}$. The TEER values were measured at selected time points $(0,10,20,30 \mathrm{~min}, 1$, $2,4,12 \mathrm{~h}$ ) during the reoxygenation period.

\section{Establishment of ischemic stroke model}

All animal experiments were carried out according to the Guide for the Care and Use of Laboratory Animals, Fudan University (Shanghai, China). Briefly, the male ICR mice weighing 19-22 g were anesthetized with $1 \%$ pentobarbital sodium solution $(0.03 \mathrm{mg} / \mathrm{kg})$ via intraperitoneal (i.p.) injection. Under an operating microscope, temporal muscle was divided and the right lateral aspect of the skull was exposed. The main trunk of the middle cerebral artery (MCA) was visualized after intravenous injection of Rose Bengal $(25 \mathrm{mg} / \mathrm{kg}$; Sigma-Aldrich, USA). A laser beam (wavelength 532 $\mathrm{nm}, 35 \mathrm{~mW}$, GL532TA-100FC, Shanghai Laser \& Optics Century, China) with $0.1 \mathrm{~mm}$ diameter was stereotactically positioned onto the MCA for $2 \mathrm{~min}$. The establishment of ischemic stroke models was confirmed by T2W-MRI at 4-6 $\mathrm{h}$ after the onset of the ischemic model.

\section{In vivo MRI studies}

In vivo MRI studies were conducted in ICR mice at $6 \mathrm{~h}$ post model establishment. All animals were anaesthetized with isoflurane (1.5-2\% in $20 \%$ oxygen) and then positioned in a 7.0 Tesla small animal MR scanner (Bruker PharmaScan, Germany). The mice were kept warm by a heating pad and their respiration rates were monitored by a Bruker Physiogard system with 20-30 breaths/min. T2W-MRI were acquired at selected time points post injection of EDV-AM or EDV-PM with the same EDV dose $(0.45 \mu \mathrm{mol} \mathrm{EDV} /$ mouse). T2W images were acquired using a fast spin echo sequence: [repetition time (TR)/echo time $(\mathrm{TE})=3000 / 36 \mathrm{~ms}, 1$ average, acquisition matrix $=256 \times 256$, field of view $=20 \times 20$ $\mathrm{mm}$, number of slices $=20$, slice thickness $=0.6 \mathrm{~mm}$, rare factor $=2$, flip angle $=180^{\circ}$. The infarct volume delineated by $\mathrm{T} 2 \mathrm{~W}$ images were quantified by using $\mathrm{NIH}$ Image J program and normalized to the volume of the whole contralateral hemisphere. The lesion volumes were corrected for the effects of brain edema by using the following equation: $\mathrm{rVL}=$ $(\mathrm{VC}-\mathrm{VI}+\mathrm{VL}) / \mathrm{VC}$, where $\mathrm{VC}=$ signal intensity of the contralateral hemispheric volumes, $\mathrm{VI}=$ signal intensity of the right-hemisphere volumes, VL = indicates uncorrected lesion volumes [44]. Diffusion tensor imaging was scanned 14 days post stroke. 30 distinct diffusion directions and five reference images were acquired by using an echo planar imaging sequence (repetition time/echo time of $5,000 / 32.2 \mathrm{~ms}$, matrix of $128 \times 128$, b-value of $1,000 \mathrm{~s} / \mathrm{mm} 2$, field of view of $16 \times 16 \mathrm{~mm}$, and slice thickness of $0.6 \mathrm{~mm}, 20$ slices). The regions of interest (ROIs) were manually drawn in the peri-infarct area on the T2W images [38]. Fractional anisotropy (FA) of DTI-MR image was derived from the tensor map with ParaVision 5.0 software (Bruker). A region of interest was delimited in the ipsilateral internal capsule (IC) of the brain. Fiber tracking was performed using TrackVis (version0.5.2.1) and Diffusion Toolkit (version 0.6.2.1) software.

\section{In vivo NIR Fluorescence Imaging}

In vivo NIR fluorescence images were obtained by Maestro in Vivo Imaging System (CRi Inc., USA) at selected time points post i.v. injection of EDV-AM or EDV-PM with the same concentration of IRB-PEG-PLA (10 nmol IRB/mouse). NIR fluorescence images were collected at a band-pass excitation filter from 745 to $785 \mathrm{~nm}$ and a long-pass emission filter over $760 \mathrm{~nm}$. At $24 \mathrm{~h}$ after administration of the micelles, the mice were sacrificed and were perfused transcardially with PBS followed by $4 \%$ paraformaldehyde (PFA). The whole brains were excised for ex vivo NIR fluorescence imaging. The regions of interest were defined and quantified by using the instrument-equipped software (Maestro software, CRi).

\section{Ex vivo HPLC studies}

EDV concentrations in ipsilateral hemisphere were determined by HPLC analysis [45]. The ischemic stroke models at $4 \mathrm{~h}$ post model establishment were injected EDV-AM or EDV-PM with a same dose of EDV (0.45 $\mu \mathrm{mol} \mathrm{EDV} /$ mouse) via i.v.. At $24 \mathrm{~h}$ post injection, the mouse models were sacrificed and their ipsilateral brain hemispheres were homogenized in ice-cold distilled water. After centrifuging $(18,000$ $\mathrm{rpm}, 10 \mathrm{~min}$ ) at $4^{\circ} \mathrm{C}$, the homogenates were collected and acetonitrile was added to precipitate proteins. The clean mixtures were centrifuged $(14,000 \mathrm{rpm}, 10$ $\mathrm{min}$ ) and the supernatants were subjected to HPLC analysis. The mobile phase was methanol/0.05 M ammonium dihydrogen phosphate solution $(\mathrm{pH}=$ 3.5) $(50: 50, \mathrm{v} / \mathrm{v})$ with isocratic mode. The wavelength of the detection was $245 \mathrm{~nm}$. HPLC chromatography of free EDV was also performed as a reference.

\section{Immunofluorescence staining}

At $24 \mathrm{~h}$ post-establishment of the ischemic stroke models, the mouse brains were excised, fixed in $4 \%$ PFA, transferred to $10 \%$ followed by $30 \%$ sucrose 
aqueous solutions for gradient dehydration. Then the brains were cryo-sectioned with a thickness of $10 \mu \mathrm{m}$. The slides were washed, permeabilized with $0.3 \%$ Triton X-100, and blocked with a 1\% BSA in PBS. Then the slides were incubated with rabbit anti-mouse $\mathrm{A}_{2 \mathrm{~A}} \mathrm{R}$ (1:200 dilution) primary antibody at $4^{\circ} \mathrm{C}$ overnight followed by goat anti-mouse CD31 (1:100 dilution) for another night. The sections were then stained with Alexa fluoro488-labeled goat anti-rabbit secondary antibody (1:500 dilution) for $2 \mathrm{~h}$ and Cruz Fluor555-labeled donkey anti-goat secondary antibody (1:200 dilution) for another $2 \mathrm{~h}$. After washing with PBS, the slides were further stained with DAPI $(0.5 \mathrm{mg} / \mathrm{mL})$ for $5 \mathrm{~min}$ and then mounted with cover slides. Similarly, the brain slides were stained with the rabbit anti-mouse caspase-3 primary antibody (1:100 dilution) followed by Alexa fluoro488-labeled donkey anti-rabbit secondary antibody (1:500 dilution) followed by DAPI (0.5 $\mathrm{mg} / \mathrm{mL}$ ). For in vitro studies, bEnd.3 cells cultured on 4-well glass bottom Lab-Tek Chamber Slide ${ }^{\mathrm{TM}}$ System (Thermo Fisher Scientific, USA) were washed, fixed, blocked, and stained with rabbit anti-mouse ZO-1 (1:100) or occludin primary antibody (1:200 dilution) followed by Alexa Fluoro488-labeled donkey anti-rabbit secondary antibody (1:500). The actin/myosin stress fibers (ASF) were stained by a rhodamine-labeled phalloidin (1:200 dilution) followed by the nucleus stain DAPI $(0.5 \mu \mathrm{g} / \mathrm{mL})$.

\section{Confocal fluorescence microscopic imaging}

The Confocal fluorescence microscopic images were collected on a Zeiss LSM 710 META confocal laser scanning microscope (Carl Zeiss, Germany). DAPI was excited with a $405 \mathrm{~nm}$ laser and the emission was detected with a photomultiplier by a 420-480 nm band-pass filter. Alexa-Flour488 was excited with a $495 \mathrm{~nm}$ laser and emission was detected by a second photomultiplier using a 505-550 nm band-pass filter. CruzFluor555 and Et were excited with a $560 \mathrm{~nm}$ laser and its emission was detected by a photomultiplier using a 570-610 nm band-pass filter. The fluorescence images were processed by ZEN 2012 software. The fluorescence intensities of the images were quantified by Image J software (NIH). All data are mean $\pm S D(n=12$ images from at least 4 mice in each group).

\section{Terminal dUTP Nick End-labeling (TUNEL) staining}

Apoptosis-associated DNA fragmentation in brain ischemia was assessed by One-Step TUNEL Assay Kit according to the manufacturer's suggestions. After 14 days treatment, the brain sections (10 $\mu \mathrm{m}$ thickness) were pre-treated with $3 \%$ citric acid to minimize the non-specific staining. After fixation, permeabilization, and treatment with DNase I for $30 \mathrm{~min}$ at $37^{\circ} \mathrm{C}$, the slides were incubated in a mixture of reaction buffer and TdT enzyme solution $(\mathrm{v} / \mathrm{v}=7 / 3)$ at $37^{\circ} \mathrm{C}$ for $1.0 \mathrm{~h}$. The slides were treated with a mixture of $10 \%(\mathrm{v} / \mathrm{v})$ fluorescein-labeled streptavidin solution and $90 \%(\mathrm{v} / \mathrm{v})$ labeling buffer at $37^{\circ} \mathrm{C}$ for $30 \mathrm{~min}$. After mounting with a glass coverslip, the slides were used for imaging studies.

\section{Imaging cerebral ROS in brain ischemia}

$200 \mu \mathrm{L}$ dihydroethidium $(1.0 \mathrm{mg} / \mathrm{mL}$ in PBS with 5\% DMSO) was injected to ischemic mouse models via i.v. at $24 \mathrm{~h}$ and $7 \mathrm{~d}$ post-ischemic model establishment (the animals had been treated with PBS, EDV-PM, free EDV or EDV-AM respectively as per protocol described in Figure 5A). At $4 \mathrm{~h}$ post HEt injection, the mice were sacrificed and perfused with PBS. The mouse brains were excised, fixed overnight in $4 \%$ formaldehyde (PFA), gradient dehydrated in $10 \%$ followed by $30 \%$ sucrose aqueous solution. Subsequently, the brains were cryo-sectioned with a thickness of $10 \mu \mathrm{m}$, incubated with DAPI $(0.5 \mathrm{mg} / \mathrm{mL})$ for $5 \mathrm{~min}$ and mounted with cover slides. The fluorescence intensity of the oxidative product ethidium (Et) was counted and normalized to the number of nuclei in the randomly selected images ( $\mathrm{n}>$ 12) from at least 3 brains by using an image analysis program (Image-Pro Plus 6.0).

\section{Functional tests}

Behavioral tests were performed before and at 1 , 4, 7 and 14 days post initial treatment by an investigator blinded to the experimental grouping. The tests consisted of a Modified Neurological Severity Score (mNSS) and a foot-fault test (Table S1). This score was derived by evaluating animals for hemiparesis (placing mice on a flat surface), abnormal movements (immobility, tremor, seizures), sensory deficits (placing, proprioception), and absent reflexes (pinna, corneal, startle). Neurological function was graded on a scale of 0-18 (normal condition: 0, mild injury: 1-6, moderate injury: 7-12 and severe injury: 13-18) [15]. In the modified foot-fault test, the forelimb placement dysfunctions of the ischemic stroke models were recorded every 5 minutes by a camera [46]. Mice were placed on elevated hexagonal grids and their paws were on the wire while moving along the grid. With each weight-bearing step, the paw may fall or slip between the wire, which was recorded as a foot fault. The total number of steps (movement of each forelimb) used to cross the grid and the number of foot faults for each forelimb were recorded [47]. 


\section{Statistical Analysis}

The experimental data were analyzed by using SPSS, version 12.0.1, for Windows (SPSS Inc., Chicago). Statistical significances between the data groups were analyzed by Mann-Whitney $U$ test. $P$ less than 0.05 was accepted as statistically significant. Numerical variables were expressed as mean \pm SD from at least three separate experiments.

\section{Supplementary Material}

Supplementary figures and tables.

http://www.thno.org/v07p0884s1.pdf

\section{Abbreviation}

EDV: edaravone; ROS: reactive oxygen species; EDV-AM: EDV encapsulated agonistic micelle; BBB: blood brain barrier; CBF: cerebral blood flow; rt-PA: Recombinant tissue plasminogen activator; CNS: central nervous system; BDNF: brain-derived neurotrophic factor; $\mathrm{A}_{2 \mathrm{~A}} \mathrm{R}$ : adenosine $2 \mathrm{~A}$ receptor; GPCRs: G-protein coupled receptors; SOD: scavenger superoxide dismutase; MRI: magnetic resonance imaging; TJs: tight junctions; NIR: near-infrared; I.H.: ipsilateral hemisphere; C.H.: contralateral hemisphere; PEG-PLA: methoxypoly (ethylene glycol)- $b$-poly (D,L-lactic acid); TEM: Transmission electron microscope; DLS: dynamic light scattering; CMC: critical micelle concentrations; ZO-1: zonula occluden-1; ASF: actin stress fiber; OGD: oxygen-glucose deprivation; TEER: transendothelial cell electrical resistance; i.v.: intravenous; $\mathrm{T} 2 \mathrm{~W}$ : T2-weighted; HEt: dihydroethidium; Et: ethidium; TUNEL: terminal-deoxynucleotidyl-transferase mediated dUTP nick end labeling; EPC: endothelial progenitor cell; DTI: diffusion tensor imaging; IC: inner capsule; FA: fractional anisotropy; mNSS: modified neurological severity score; FBS: fetal bovine serum; PS: penicillin and streptomycin; ATCC: American Type Culture Collection; $\mathrm{CO}_{2}$ : carbon dioxide; i.p.: intraperitoneal; ROIs: region of interests; DMEM: Dulbecco's modified Eagle's medium.

\section{Acknowledgements}

This work was supported by the National Basic Research Program of China (973 Program, 2013CB733801, 2013CB733802), the National Natural Science Foundation of China (Nos. 81571741, 81371624, 81525014, 81371538), the Shanghai Foundation for Development of Science and Technology (Nos. 15140901300), “Shuguang Program" supported by Shanghai Education Development Foundation and Shanghai Municipal Education Commission. We thank the helpful discussion with Dr. Charlotte Rivas from Imperial
College, London, UK. Qu Jin and Yu Cai contributed equally to this work.

\section{Competing Interests}

The authors have declared that no competing interest exists.

\section{References}

1. Allen CL, Bayraktutan U. Oxidative stress and its role in the pathogenesis of ischaemic stroke. Int J Stroke. 2009; 4: 461-70.

2. Adeoye $\mathrm{O}$, Hornung $\mathrm{R}$, Khatri $\mathrm{P}$, et al. Recombinant tissue-type plasminogen activator use for ischemic stroke in the United States: a doubling of treatment rates over the course of 5 years. Stroke. 2011; 42: 1952-5.

3. Hacke $W$, Kaste $M$, Fieschi $C$, et al. Intravenous thrombolysis with recombinant tissue plasminogen activator for acute hemispheric stroke. The European Cooperative Acute Stroke Study (ECASS). JAMA. 1995; 274: 1017-25.

4. Yamaguchi T, Mori E, Minematsu $\mathrm{K}$, et al. Alteplase at $0.6 \mathrm{mg} / \mathrm{kg}$ for acute ischemic stroke within 3 hours of onset: Japan Alteplase Clinical Trial (J-ACT). Stroke. 2006; 37: 1810-5.

5. Fiorelli M, Bastianello $S$, von Kummer R, et al. Hemorrhagic transformation within 36 hours of a cerebral infarct relationships with early clinical deterioration and 3-month outcome in the European Cooperative Acute Stroke Study I (ECASS I) cohort. Stroke. 1999; 30: 2280-4.

6. Kim CK, Kim T, Choi IY, et al. Ceria nanoparticles that can protect against ischemic stroke. Angew Chem Int Ed Engl. 2012; 51: 11039-43.

7. Cherubini A, Ruggiero C, Polidori MC, et al. Potential markers of oxidative stress in stroke. Free Radic Biol Med. 2005; 39: 841-52.

8. Lubos E, Handy DE, Loscalzo J. Role of oxidative stress and nitric oxide in atherothrombosis. Front Biosci. 2008; 13: 5323-44.

9. Amantea D, Nappi G, Bernardi G, et al. Post-ischemic brain damage: pathophysiology and role of inflammatory mediators. FEBS J. 2009; 276: 13-26.

10. Banno M, Mizuno T, Kato H, et al. The radical scavenger edaravone prevents oxidative neurotoxicity induced by peroxynitrite and activated microglia. Neuropharmacology. 2005; 48: 283-90.

11. Zhang N, Komine-Kobayashi M, Tanaka R, et al. Edaravone reduces early accumulation of oxidative products and sequential inflammatory responses after transient focal ischemia in mice brain. Stroke. $2005 ; 36: 2220-5$.

12. Kikuchi K, Kawahara K, Miyagi N, et al. Edaravone: a new therapeutic approach for the treatment of acute stroke. Med Hypotheses. 2010; 75: 583-5.

13. Otomo E, Tohgi $\mathrm{H}$, Kogure $\mathrm{K}$, et al. Effect of a novel free radical scavenger, edaravone (MCI-186), on acute brain infarction - Randomized, placebo-controlled, double-blind study at multicenters. Cerebrovasc Dis. 2003; 15: 222-9.

14. Higashi $\mathrm{Y}$, Jitsuiki $\mathrm{D}$, Chayama $\mathrm{K}$, et al Edaravone (3-methyl-1-phenyl-2-pyrazolin-5-one), a novel free radical scavenger, for treatment of cardiovascular diseases. Recent Pat Cardiovasc Drug Discov. 2006; $1: 85-93$

15. Wu HY, Tang Y, Gao LY, et al. The synergetic effect of edaravone and borneol in the rat model of ischemic stroke. Eur J Pharmacol. 2014; 740: 522-31.

16. Yu B, Ruan M, Dong X, et al. The mechanism of the opening of the blood-brain barrier by borneol: A pharmacodynamics and pharmacokinetics combination study. J Ethnopharmacol. 2013.

17. Shimazaki H, Hironaka K, Fujisawa T, et al. Edaravone-loaded liposome eyedrops protect against light-induced retinal damage in mice. Invest Ophthalmol Vis Sci. 2011; 52: 7289-97.

18. Singhal A, Morris VB, Labhasetwar V, et al. Nanoparticle-mediated catalase delivery protects human neurons from oxidative stress. Cell Death Dis. 2013; 4. e903.

19. Krol S, Macrez R, Docagne F, et al. Therapeutic benefits from nanoparticles: the potential significance of nanoscience in diseases with compromise to the blood brain barrier. Chem Rev. 2013; 113: 1877-903.

20. Zlokovic BV. The blood-brain barrier in health and chronic neurodegenerative disorders. Neuron. 2008; 57: 178-201.

21. Gao X, Qian J, Zheng S, et al. Overcoming the blood-brain barrier for delivering drugs into the brain by using adenosine receptor nanoagonist. ACS Nano. 2014; 8: 3678-89

22. Zheng S, Bai YY, Liu $Y$, et al. Salvaging brain ischemia by increasing neuroprotectant uptake via nanoagonist mediated blood brain barrier permeability enhancement. Biomaterials. 2015; 66: 9-20.

23. Sandoval KE, Witt KA. Blood-brain barrier tight junction permeability and ischemic stroke. Neurobiol Dis. 2008; 32: 200-19.

24. Gao X, Li C. Nanoprobes visualizing gliomas by crossing the blood brain tumor barrier. Small. 2014; 10: 426-40.

25. de Gracia Lux C, Joshi-Barr S, Nguyen T, et al. Biocompatible polymeric nanoparticles degrade and release cargo in response to biologically relevant levels of hydrogen peroxide. J Am Chem Soc. 2012; 134: 15758-64.

26. Lim WT, Tan EH, Toh CK, et al. Phase I pharmacokinetic study of a weekly liposomal paclitaxel formulation (Genexol-PM) in patients with solid tumors. Ann Oncol. 2010; 21: 382-8. 
27. de Lera Ruiz M, Lim YH, Zheng J. Adenosine A2A receptor as a drug discovery target. J Med Chem. 2014; 57: 3623-50.

28. Yan H, Wang L, Wang J, et al. Two-order targeted brain tumor imaging by using an optical/paramagnetic nanoprobe across the blood brain barrier. ACS Nano. 2012; 6: 410-20.

29. Han SK, Na K, Bae YH. Sulfonamide based pH-sensitive polymeric micelles: physicochemical characteristics and $\mathrm{pH}$-dependent aggregation. Colloids Surf A, Physicochem Eng Asp. 2003; 214: 49-59.

30. Kasperczyk A, Slowinska-Lozynska L, Dobrakowski M, et al. The effect of lead-induced oxidative stress on blood viscosity and rheological properties of erythrocytes in lead exposed humans. Clin Hemorheol Microcirc. 2014; 56: 187-95.

31. Gibson CL, Srivastava K, Sprigg N, et al. Inhibition of Rho-kinase protects cerebral barrier from ischaemia-evoked injury through modulations of endothelial cell oxidative stress and tight junctions. J Neurochem. 2014; 129: 816-26.

32. Kim GW, Sugawara $\mathrm{T}$, Chan PH. Involvement of oxidative stress and caspase-3 in cortical infarction after photothrombotic ischemia in mice. J Cereb Blood Flow Metab. 2000; 20: 1690-701.

33. Nicholson DW, Thornberry NA. Caspases: killer proteases. Trends Biochem Sci. 1997; 22: 299-306

34. Manabat $\mathrm{C}$, Han $\mathrm{BH}$, Wendland $\mathrm{M}$, et al. Reperfusion differentially induces caspase- 3 activation in ischemic core and penumbra after stroke in immature brain. Stroke. 2003; 34: 207-13.

35. Gao X, Wang YC, Liu Y, et al. Nanoagonist-mediated endothelial tight junction opening: A strategy for safely increasing brain drug delivery in mice. J Cereb Blood Flow Metab. 2016.

36. Balkaya M, Krober JM, Rex A, et al. Assessing post-stroke behavior in mouse models of focal ischemia. J Cereb Blood Flow Metab. 2013; 33: 330-8.

37. Miyazaki T, Kimura $\mathrm{Y}$, Ohata $\mathrm{H}$, et al. Distinct effects of tissue-type plasminogen activator and SMTP-7 on cerebrovascular inflammation following thrombolytic reperfusion. Stroke. 2011; 42: 1097-104.

38. Bai YY, Wang L, Chang D, et al. Synergistic Effects of Transplanted Endothelial Progenitor Cells and RWJ 67657 in Diabetic Ischemic Stroke Models. Stroke. 2015; 46: 1938-46.

39. Berkhemer OA, Fransen PS, Beumer D, et al. A randomized trial of intraarterial treatment for acute ischemic stroke. N Engl J Med. 2015; 372: 11-20.

40. Huile G, Shuaiqi P, Zhi Y, et al. A cascade targeting strategy for brain neuroglial cells employing nanoparticles modified with angiopep-2 peptide and EGFP-EGF1 protein. Biomaterials. 2011; 32: 8669-75.

41. Yu Z, Liu J, Guo S, et al. Neuroglobin-overexpression alters hypoxic response gene expression in primary neuron culture following oxygen glucose deprivation. Neuroscience. 2009; 162: 396-403.

42. Hseu YC, Chou CW, Senthil Kumar KJ, et al. Ellagic acid protects human keratinocyte $(\mathrm{HaCaT})$ cells against UVA-induced oxidative stress and apoptosis through the upregulation of the HO-1 and Nrf-2 antioxidant genes. Food Chem Toxicol. 2012; 50: 1245-55.

43. Yang HL, Lin SW, Lee CC, et al. Induction of Nrf2-mediated genes by Antrodia salmonea inhibits ROS generation and inflammatory effects in lipopolysaccharide-stimulated RAW264.7 macrophages. Food Funct. 2015; 6: 230-41.

44. Qian C, Li PC, Jiao Y, et al. Precise Characterization of the Penumbra Revealed by MRI: A Modified Photothrombotic Stroke Model Study. PLoS One. 2016; 11: e0153756.

45. Wan X, Zheng X, Pang X, et al. The potential use of lapatinib-loaded human serum albumin nanoparticles in the treatment of triple-negative breast cancer. Int J Pharm. 2015; 484: 16-28.

46. Hernandez TD, Schallert T. Seizures and recovery from experimental brain damage. Exp Neurol. 1988; 102: 318-24.

47. Zhang L, Schallert $\mathrm{T}$, Zhang ZG, et al. A test for detecting long-term sensorimotor dysfunction in the mouse after focal cerebral ischemia. J Neurosci Methods. 2002; 117: 207-14. 